\title{
Genes Expressed after Retinoic Acid-Mediated Differentiation of Embryoid Bodies Are Likely to Be Expressed during Embryo Development
}

\author{
Srećko Gajović,*`† Kamal Chowdhury,* and Peter Gruss*,1
}

\begin{abstract}
*Department of Molecular Cell Biology, Max Planck Institute of Biophysical Chemistry, Am Fassberg 11, P.O. Box 2841, 37018, Göttingen, Germany; and tDepartment of Histology and Embryology, School of Medicine, University of Zagreb, Zagreb, Croatia
\end{abstract}

\begin{abstract}
In order to test if retinoic acid-mediated differentiation of embryoid bodies can be used as an in vitro preselection method for ES cell lines generated by gene trap, we correlated gene expression after in vitro differentiation and in 11.5-day embryos. Fifty-two genes captured by gene trap and expressed in undifferentiated embryonic stem cells were analyzed. Most genes expressed after differentiation in vitro were also expressed during embryo development. In order to correlate the expression patterns in vitro and in vivo, the in vitro expression in the center and in the periphery of the embryoid body outgrowths was observed. This allowed us to distinguish, according to in vitro expression, not expressed genes from those expressed widely in 11.5-day embryos. Consequently, with this parameter we increased the probability to obtain the restricted expression patterns in vivo. This study demonstrates the potential of the differentiation procedure in combination with the gene trap to select in vitro for genes expressed during embryo development.
\end{abstract} (c) 1998 Academic Press

Key Words: retinoic acid; embryonic stem cells; embryoid bodies; gene trap.

\section{INTRODUCTION}

Embryonic stem (ES) cells posses an extraordinary ability to contribute to embryo development and to generate, via germ cells, a complete animal [1]. Consequently, ES cells are genetically modified in culture, and animals carrying this genetic modification are generated. This technique is mainly used for targeted mutagenesis by homologous recombination [2]. In contrast to this spedific approach, the ES cell genome can be randomly altered by inserting a nonhomologous construct, a method referred as gene trap [3, 4]. In this way a previously unknown gene can be tagged and subsequently analyzed [5]. However, not every trapped gene will be interesting

\footnotetext{
${ }^{1}$ To whom correspondence and reprint requests should be addressed. Fax: ++49551 2011504. E-mail: pgruss@gwdg.de.
}

and therefore the procedure includes a tedious selection process. The predominant rationale is that the genes expressed in a restricted manner during embryo development are more likely to have a control function, and, when mutated, to generate changes of phenotype. This implies selection of gene trap insertions after producing a chimeric animal and a subsequent analysis of the expression pattern of the trapped gene in vivo [6]. As this requires vast resources and number of animals, a substantial effort is being made to modify the random screen in order to get genes of particular interest. One of the possible solutions, termed "in vitro preselection," attempts by analyzing in vitro differentiated ES cells to predict genes expressed in corresponding differentiated tissues in vivo $[7,8]$.

In order to test if the retinoic acid (RA)-mediated differentiation of ES-cell-derived embryoid bodies can be used as an in vitro preselection method for gene trap, we performed a large scale screen comparing the gene expression patterns in vitro and in vivo. The applied differentiation procedure has already been shown to generate neurons and glia and to mediate the expression of Pax6, a developmentally regulated transcription factor expressed during central nervous system development [9-11]. We demonstrated that most of the genes expressed in vitro were also expressed during embryo development. However, there was no specific enrichment of genes expressed in the central nervous system (CNS).

\section{MATERIALS AND METHODS}

Cell culture and generation of the gene trap clones. MPI-II ES cells [12] or R1 ES cells [13] were grown on mitomycin C (100 $\mu \mathrm{g} / \mathrm{ml}$, Sigma, St. Louis, MO)-treated primary embryonic fibroblasts in Dulbecco's modified Eagle's medium (DMEM $4.5 \mathrm{~g}$ glucose/l, GIBCO BRL, Gaithersburg, MD) supplemented with $1 \mathrm{mM}$ sodium pyruvate (GIBCO BRL, Gaithersburg, MD), 1\% of stock solution of nonessential amino acids (GIBCO BRL, Gaithersburg, MD), $2 \mathrm{mM}$ L-glutamine, $100 \mu \mathrm{mol} \beta$-mercaptoethanol, $500 \mathrm{U} / \mathrm{ml}$ leukemia inhibitory factor (LIF, supplied by Amgene, Newbury Park, CA), and $20 \%$ heat-inactivated fetal calf serum (FCS, selected batch from GIBCO BRL, Gaithersburg, MD). Cells were regularly split every 2 days. 
Two gene trap vectors: pGT1.8geo (kindly provided by W. Skarnes [14]) and pKC199 $\beta$ geo [15] were used. They contained the splice acceptor sequence from mouse En-2 or Hoxc9 gene fused to the promoterless $\beta$ geo, which is a lacZ-neomycin phosphotransferase fusion gene [4]. After electroporation and selection for 10 days by 250 $\mu \mathrm{g} / \mathrm{ml} \mathrm{G} 418$ (GIBCO BRL, Gaithersburg, MD), resistant clones were isolated and stained with X-gal for the presence of $\beta$-galactosidase. The positive clones were expanded and used for further analysis.

In vitro differentiation. In vitro differentiation of ES cells in presence of RA and low level (1\%) FCS was performed as described previously [11]. ES cell clones showing $\beta$-galactosidase activity in undifferentiated conditions were seeded onto a gelatin (0.1\%)-coated tissue culture dish. Cells were kept in full ES cell medium as described above. After 2 days the cells were recovered by gentle trypsinization (0.05\% trypsin/EDTA solution) and cultured in suspension for embryoid body preparation in DMEM medium with $1 \%$ FCS. The embryoid bodies were treated for next 4 days with $1 \mu \mathrm{M}$ all trans-RA (Sigma, St. Louis, MO) prepared from a stock of $10 \mathrm{mM}$ RA in ethanol kept at $-20^{\circ} \mathrm{C}$ in the dark. After 4 days of the suspension culture the embryoid bodies were transferred to gelatin-coated cell culture dishes with medium lacking RA, cultured for 5 days, and stained with X-gal.

Production and analysis of chimeras. Chimeras were produced by morula aggregation [12, 13]. Eight cell stage embryos (2.5-day embryos; day of the plug $=0.5$ ) were recovered from superovulated and mated NMRI or CD1 females. The zona pellucida was removed and embryos were placed in a drop of M16 medium together with a clump of an average of $10 \mathrm{ES}$ cells for overnight culture. The resulting blastocysts were transferred into the uterine horn of pseudopregnant recipient females mated with vasectomized males 2.5 days before. 11.5-day chimeric embryos were recovered and stained in toto with $\mathrm{X}$-gal. The degree of chimerism was assessed by the pigmentation of the eye, and for all clones more than one chimeric embryo was analyzed.

X-gal staining. Cultured cells or whole embryos were fixed in a mixture of $2 \%$ formal dehyde and $0.2 \%$ glutaraldehyde in phosphatebuffered saline (PBS), rinsed 3 times in PBS, and incubated overnight at $37^{\circ} \mathrm{C}$ in a mixture of $1 \mathrm{mg} / \mathrm{ml} \mathrm{X}$-gal, $10 \mathrm{mM} \mathrm{K}{ }_{3} \mathrm{Fe}(\mathrm{CN})_{6}, 10$ $\mathrm{mM} \mathrm{K}{ }_{4} \mathrm{Fe}(\mathrm{CN})_{6}$, and $2 \mathrm{mM} \mathrm{MgCl}_{2}$ in PBS. After incubation specimens were washed in PBS for $24 \mathrm{~h}$ and then cleared in ascending concentrations of glycerol in PBS up to $75 \%$.

Statistical analysis. The correlation between gene expression in vitro and in vivo was determined using the nonparametric McNemare's and Stewart's test for dependent samples. The concordance between two procedures was present if the differences between them were shown to be random ( $P>0.05)$.

\section{RESULTS}

To determine a correlation between gene expression in vitro, after RA-mediated embryoid body differentiation, and in vivo, during embryo development, 52 ES cell clones carrying gene trap insertion within the genes expressed in undifferentiated ES cells were analyzed. All of these clones were neomycin resistant and had $\beta$-galactosidase activity in undifferentiated cells. Each clone was simultaneously processed for in vitro differentiation procedure and for morula aggregation.

To preselect in vitro for genes expressed in the nervous system, ES cells were differentiated by culturing them in suspension in presence of $1 \mu \mathrm{M}$ RA and low level (1\%) fetal calf serum. After 4 days of culture, resulting embryoid bodies were plated to the adhesive surface in the absence of RA. After attachment the embryoid bodies gave rise to the colonies of differentiated cells consisting of a central clump and a monolayer of peripheral cells. Neuronal processes were visible extending from the central clumps toward the periphery (Figs. $1 \mathrm{~A}$ and $1 \mathrm{C}$ ).

The expression of genes captured by gene trap was determined by X-gal staining of the embryoid body outgrowths 5 days after attachment. This was compared to the gene expression in the corresponding 11.5-day chimeric embryos, determined by whole mount X-gal staining. Three main parameters were: ubiquitous gene expression, restricted expression, and no expression (Table 1A). After in vitro differentiation $14 \%$ of genes had ubiquitous expression, $48 \%$ restricted, and $38 \%$ showed no expression. In 11.5-day embryos corresponding genes were in $30 \%$ of cases ubiquitously expressed, 35\% re stricted, and 35\% were not expressed.

When in vitro and in vivo gene expression was compared, only 2 of 32 genes expressed after in vitro differentiation were not expressed in 11.5-day embryos. This concordance between in vitro and in vivo system was statistically significant (differences $P>$ 0.05 ; Table 1A). All genes ubiquitously expressed in vitro, were also ubiquitous in vivo. However, not all of the genes with restricted expression in vitro were restricted in vivo. Nine of them were ubiquitously expressed in 11.5-day embryos (Fig. 1A), and two of them were not expressed at all. These differences between two systems were statistically significant $(P<0.01$, Table $1 A)$. Presuming that in vitro differentiation of embryoid body outgrowths proceeds from the periphery toward the center, and that the less differentiated cells are present in the centre of the outgrowths, we set apart the genes expressed only in the central clumps from those expressed in the periphery as well (Table 1B; Figs. 1A-1C). When expression patterns were classified in this way, it was possible to distinguish, according to in vitro expression, the genes expressed ubiquitously in vivo from those not expressed at all. The concordance between in vitro and in vivo expression was statistically significant (differences $P>0.05$ ). The genes with restricted expression pattern in vivo showed three possibilities in vitro: restricted expression in the central clumps and in the periphery, restricted expression only in the central clumps, and no expression. The highest number of clones belonged to the "central clumps only" category (Fig. 1B).

To test the likelihood of in vitro preselection for genes expressed during central nervous system (CNS) development, the restricted expression patterns in 11.5-day embryos were classified in two groups: patterns including the CNS and those not including CNS (Table 1C; Figs. 1D and 1E). From 18 genes expressed in restricted manner in 11.5-day 


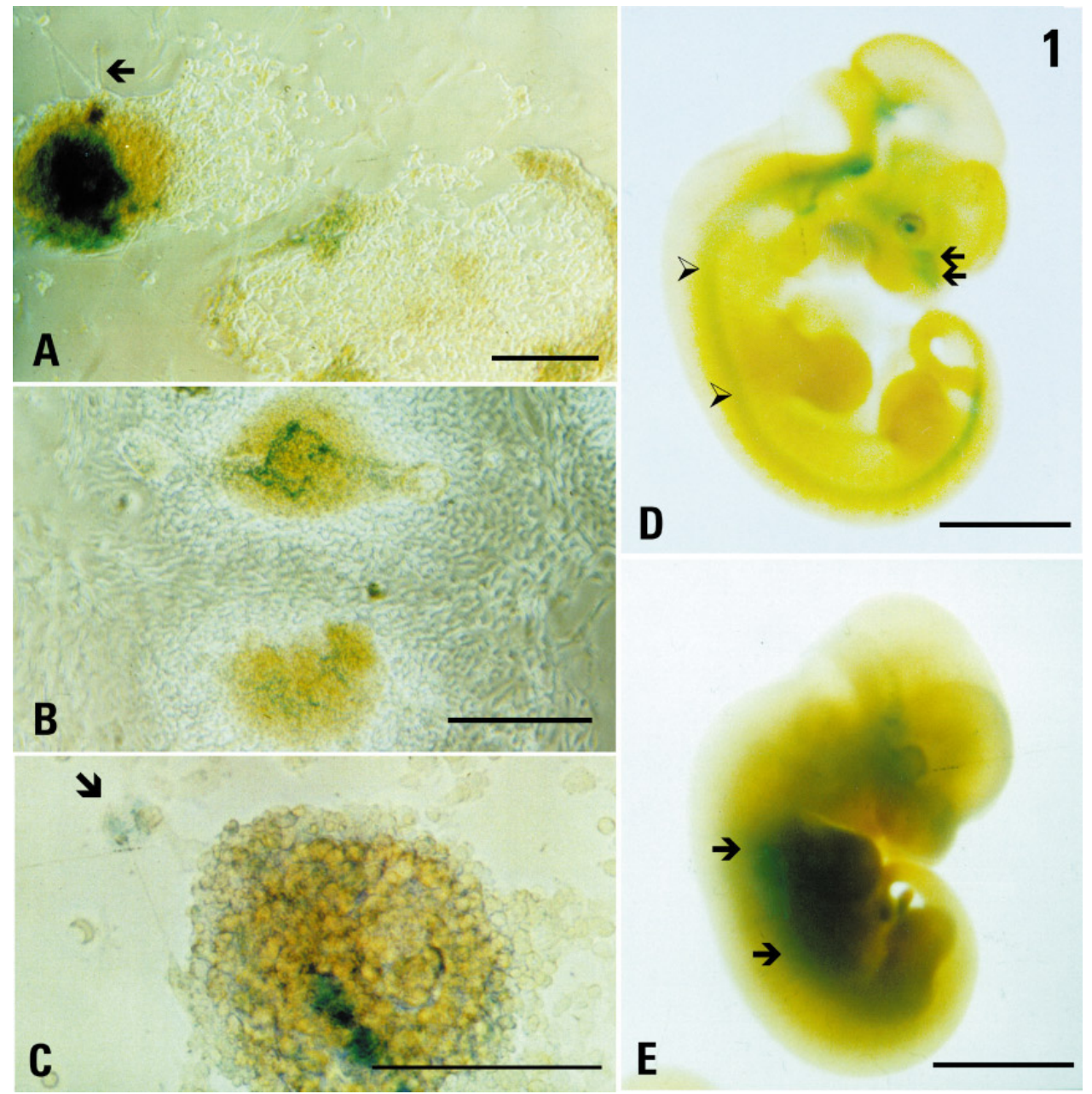

FIG. 1. (A-C) Embryoid body outgrowths after differentiation stained by X-gal; bar $100 \mu \mathrm{m}$. (D, E) 11.5-day embryos stained by X-gal; bar 1 $\mathrm{mm}$. (A) In vitro expression of a gene, which is ubiquitously expressed in 11.5-day embryos. The expression is located within the central dump and the periphery of the embryoid body outgrowth; arrow, neurite outgrowths. (B) In vitro expression of a gene, which is expressed in 11.5 -day embryos along the neural tube. The expression is confined to the restricted portions of the central clumps. (C, D) In vitro and in vivo expression of the same gene. In vitro expression is located in the central dumps and in the outgrowth periphery. Gene was expressed in the neuron-like cells with long cell process (arrows). The expression in 11.5-day embryos is located along the neural tube (arrowheads) and in the nasal region (double arrows). (E) The expression pattern in the mesenchymal structures inside the embryo (arrows) not involving CNS. In vitro expression of the same gene was present in restricted regions of the central clumps and the periphery of the embryoid body outgrowths.

embryos, two-thirds were expressed in CNS. When compared with their expression after differentiation in vitro, the genes were distributed through in vitro categories regardless to their expression in the CNS (Table 1C). The morphologically distinct neural cell processes were present after differentiation, but only two genes were expressed in cells having the processes. These two genes were expressed in the CNS in vivo, what we confirmed as well on tissue sections (Figs. 1C and 1D).

\section{DISCUSSION}

The RA-mediated differentiation of embryoid bodies generates from pluripotent ES cells a sizable percentage of cells of the nervous tissue [9]. The resulting neurons exhibit a number of neuronal markers and possess the polarity and functional synaptic transmission typical of CNS neurons [16, 17]. Other cell types, without morphologies as distinctive as neuronal cells, differentiate as well. In this study it was shown that genes expressed in different regions of the embryo were expressed as well upon RA-mediated embryoid body differentiation. This implies that RA does not induce only neuronal genes in vitro, but its effect is more general. However, this conclusion is based on investigation of a sample from a predefined group of genes, i.e., genes expressed in undifferentiated ES cells. Thus all investigated genes were expressed already before differentiation, 
TABLE 1

Relationships between Gene Expression in Vitro and in Vivo

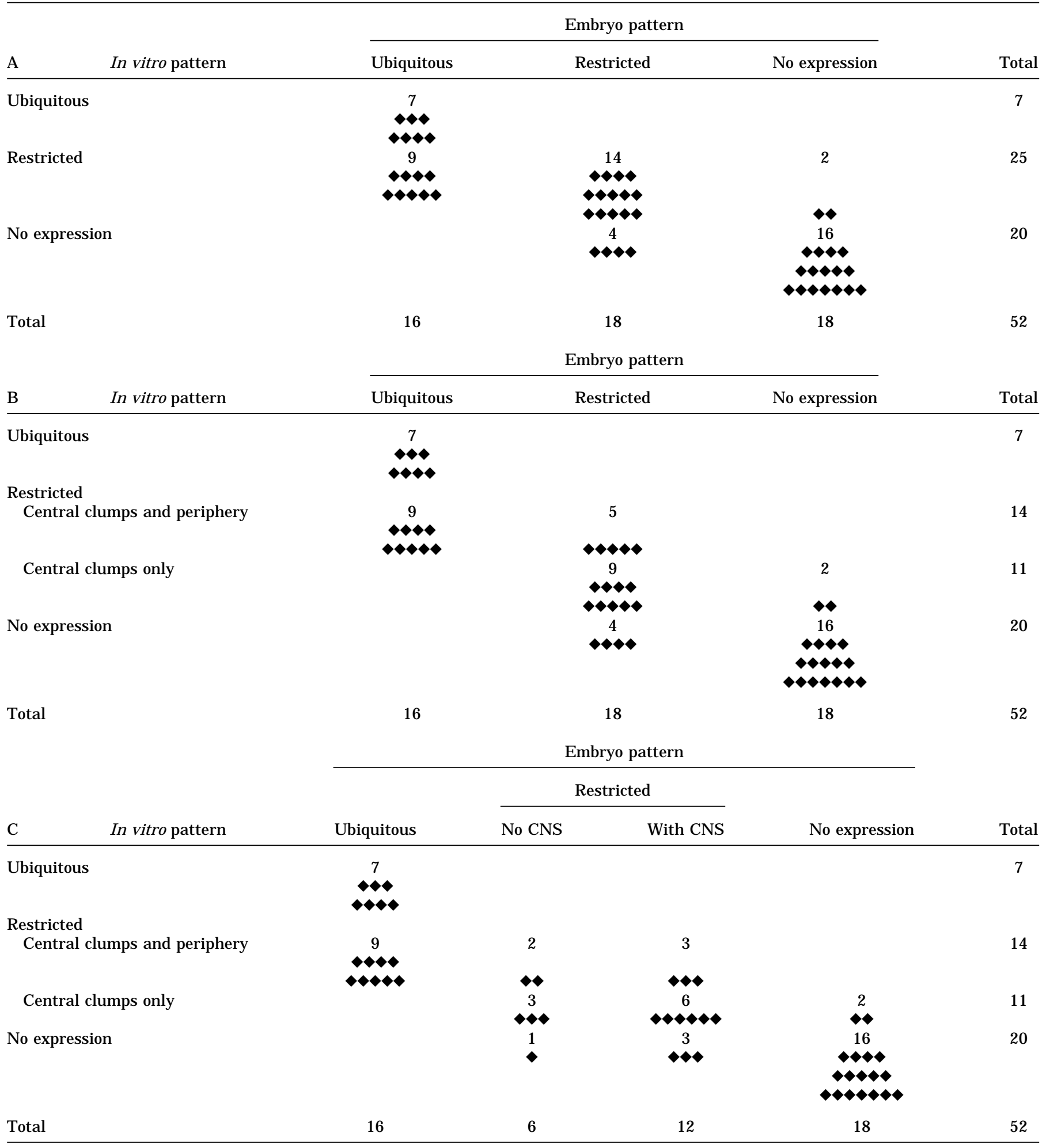

Note. Relationships between gene expression after RA-mediated differentiation of embryoid bodies and in the 11.5-day embryos. (A) Gene expression is divided in three categories: ubiquitous expression, restricted expression, and genes not expressed. If ubiquitous and restricted categories (four upper left fields) are combined together (i.e., expressed genes), there is a concordance between in vitro and in vivo system $\left(\chi^{2}=0.66, \mathrm{df}=1, \mathrm{P}>0.05\right)$. For the whole table, differences between these two systems are statistically significant $\left(\chi^{2}=9.66, \mathrm{df}=2, \mathrm{P}<\right.$ 0.01). (B) The same table with the restricted expressions in vitro divided in two categories: genes expressed in both the central clumps and in the periphery of the embryoid body outgrowths, and those expressed only in the central clumps. (C) The previous table, but the embryo patterns divided into those involving and not involving CNS $\left(\chi^{2}=0.98, \mathrm{df}=3, \mathrm{P}>0.05\right)$. 
and no genes specifically activated by the treatment could be taken into account. Consequently, it is possible to conclude that RA induces in vitro the expression of genes expressed in vivo in nonneural as well as in neural tissues. The genes not expressed in 11.5-day embryos are mostly turned off in vitro.

It is obvious that the applied differentiation procedure used together with the gene trap could not exclusively select for genes expressed in a restricted manner during embryo development. However, it could substantially increase the probability to obtain such genes. The comparable efficiency was achieved by an alternative strategy termed "induction gene trap screen," using RA treatment of undifferentiated ES cells in order to select genes according to the responsiveness to RA [18, 19]. Our approach differs from the "induction screen" as it attempts to predict the in vivo gene expression according to the expression pattern in differentiated cells. As a consequence, most genes not expressed after in vitro differentiation procedure were as well not expressed in embryo, contrary to the genes repressed by RA during the "induction screen," which showed the restricted expression pattern in embryo [18]. The final gene trap strategy will depend on a specific task of the particular investigator. It is certainly advisable to combine in vitro differentiation procedure with molecular characterization of the trapped gene and the construct integration [8, 20, 21]. An additional approach is to analyze the coexpression of specific markers with the trapped genes [7, 8]. We checked for genes expressed in cells with characteristic neuron-like morphology and found two genes, which were indeed expressed in the developing nervous system.

The main advantage of the proposed differentiation procedure is that it is rather simple and therefore can be applied to high number of ES cell clones. One can even rely on simple monitoring of the gradient of differentiation from the central clump to the periphery of the embryoid body outgrowths. This can be used in order to improve the current gene trap approach in which only genes expressed in undifferentiated ES cells are trapped. The RA-mediated differentiation of embryoid bodies can be combined with gene trap procedure where the neomycin resistance will be driven by a constitutive promoter. This would allow the screening for genes not expressed in undifferentiated ES cells, but expressed specifically after differentiation, a route which should target a new group of developmentally important genes.

The authors thank Dr. Andras Nagy for providing R1 ES cells, Dr. Anne K. Voss and Dr. Tim Thomas for providing MPI-II ES cells and help in gene trap, and Dr. William C. Skarnes for providing
pGT1.8geo. We also thank Dr. Silvije Vuletic for help with statistics, Dr. Kenneth Ewan for reading the manuscript, Rebecca Emeny, Marion Stäger, Victor Diaz Salamanca, and Gudrun Weinrich for technical assistance, and AmGen Inc. for financial support. S.G. was supported by Max-Planck and EMBO fellowships.

\section{REFERENCES}

1. Bradley, A., Evans, M., Kaufman, M. H., and Robertson, E. (1984). F ormation of germ-line chimaeras from embryo-derived teratocarcinoma cell lines. Nature 309, 255-256.

2. Mansour, S. L., Thomas, K. R., and Capecchi, M. R. (1988). Disruption of the proto-oncogene int- 2 in mouse embryo-derived stem cells: A general strategy for targeting mutations of nonselectable genes. Nature 336, 348-352.

3. Gossler, A., J oyner, A. L., Rossant, J ., and Skarnes, W. C. (1989). Mouse embryonic stem cells and reporter constructs to detect devel opmentally regulated genes. Science 244, 463- 465.

4. Friedrich, G., and Soriano, P. (1991). Promoter traps in embryonic stem cells: A genetic screen to identify and mutate developmental genes in mice. Genes Dev. 5, 1513-1523.

5. Chowdhury, K., Bonaldo, P., Torres, M., Stoykova, A., and Gruss, P. (1997). Evidence for the stochastic integration of gene trap vectors into the mouse germ line. Nucleic Acids Res. 25, 1531-1536.

6. Wurst, W., Rossant, J ., Prideaux, V., Kownacka, M., J oyner, A., Hill, D. P., Guillemot, F., Gasca, S., Cado, D., Auerbach, A., and Ang, S.-L. (1995). A large scale gene-trap screen for insertional mutations in developmentally regulated genes in mice. Genetics 139, 889- 899.

7. Shirai, M., Miyashita, A., Ishii, N., Itoh, Y., Satokata, I., Watanabe, Y. G., and Kuwano, R. (1996). A gene trap strategy for identifying the gene expressed in the embryonic nervous system. Zool. Sci. 13, 277-283.

8. Baker, R. K., Haendel, M. A., Swanson, B. J ., Shambaugh, J . C., Micales, B. K., and Lyons, G. E. (1997). In vitro preselection of gene-trapped embryonic stem cell clones for characterizing novel developmentally regulated genes in the mouse. Dev. Biol. 185, 201-214.

9. Bain, G., Kitchens, D., Yao, M., Huettner, J . E., and Gottlieb, D. I. (1995). Embryonic stem cells express neuronal properties in vitro. Dev. Biol. 168, 342-357.

10. Fraichard, A., Chassande, O., Bilbaut, G., Dehay, C., Savatier, P., and Samarut, J . (1995). In vitro differentiation of embryonic stem cells into glial cells and functional neurons. J . Cell. Sci. 108, 3181-3188.

11. Gajović, S., St-Onge, L., Yokota, Y., and Gruss, P. (1997). Retinoic acid mediates Pax6 expression during in vitro differentiation of embryonic stem (ES) cells. Differentiation 62, 187-192.

12. Voss, A. K., Thomas, T., and Gruss, P. (1997). Germ line chimeras from female ES cells. Exp. Cell Res. 230, 45- 49.

13. Nagy, A., Rossant, J ., Nagy, R., Abramow-Newerly, W., and Roder, J. C. (1993). Derivation of completely cell culture-derived mice from early-passage embryonic stem cells. Proc. Natl. Acad. Sci. USA 90, 8424-8428.

14. Skarnes, W. C., Auerbach, B. A., and J oyner, A. L. (1992). A gene trap approach in mouse embryonic stem cells: The lac $Z$ reporter is activated by splicing, reflects endogenous gene expression and is mutagenic in mice. Genes Dev. 6, 903-918.

15. Thomas, T., Voss, A. K., Chowdhury, K., and Gruss, P. (1997). A strategy for selecting gene trap insertion events in the $5^{\prime}$ end of genes, in preparation.

16. Strübing, K., Ahnert-Hilger, G., Shan, J., Wiedenmann, B., Hescheler, J ., and Wobus, A. M. (1995). Differentiation of plu- 
ripotent embryonic stem cells into neuronal lineage in vitro gives rise to mature inhibitory and excitatory neurons. Mech. Dev. 53, 275-287.

17. Finley, M. F. A., Kulkarni, N., and Huettner, J. E. (1996). Synapse formation and establishment of neuronal polarity by P19 embryonic carcinoma cells and embryonic stem cells. J . Neurosci. 16, 1056-1065.

18. Forrester, L. M., Nagy, A., Sam, M., Watt, A., Stevenson, L., Bernstein, A., J oyner, A. L., and Wurst, W. (1996). An induction gene trap screen in embryonic stem cells: I dentification of genes that respond to retinoic acid in vitro. Proc. Natl. Acad. Sci. USA 93, 1677-1682.

\section{Received December 17, 1997}

Revised version received February 5, 1998
19. Sam, M., Wurst, W., Forrester, L., Vauti, F., Heng, H., and Bernstein, A. (1996). A novel family of repeat sequences in the mouse genome responsive to retinoic acid. Mamm. Genome 7, 741-748.

20. Holzschu, D., Lapierre, L., Neubaum, D., and Mark, W. H. (1997). A molecular strategy for the rapid screening of gene traps based on sequence identity and gene expression pattern in adult mice. Transgenic Res. 6, 97-106.

21. Townley, D. J., Avery, B. J ., Rosen, B., and Skarnes, W. C. (1997). Rapid sequence analysis of gene trap integrations to generate a resource of insertional mutations in mice. Genome Res. 7, 293-298. 